\title{
EGFR, PIK3CA, KRAS and BRAF mutations in meningiomas
}

\author{
MATEUSZ BUJKO $^{1}$, PAULINA KOBER ${ }^{1}$, ANDRZEJ TYSAROWSKI $^{1}$, EWA MATYJA $^{2}$, \\ TOMASZ MANDAT $^{3}$, WIESŁAW BONICKI ${ }^{3}$ and JANUSZ ALEKSANDER SIEDLECKI ${ }^{1}$ \\ ${ }^{1}$ Department of Molecular and Translational Oncology, Maria Sklodowska-Curie Memorial Cancer Center \\ and Institute of Oncology, Warsaw 02-781; ${ }^{2}$ Department of Experimental and Clinical Neuropathology, \\ M. Mossakowski Medical Research Centre, Polish Academy of Sciences, Warsaw 02-106; ${ }^{3}$ Department of \\ Neurosurgery, Maria Sklodowska-Curie Memorial Cancer Center and Institute of Oncology, Warsaw 02-781, Poland
}

Received August 12, 2013; Accepted February 11, 2014

DOI: $10.3892 / 01.2014 .2042$

\begin{abstract}
Meningiomas are among the most frequent intracranial tumors. Treatment involves surgical resection with optional subsequent radiotherapy for high-grade meningiomas or radiosurgery following incomplete tumor removal. At present, no pharmacological agents are used as treatment. The use of targeted therapies has been considered, and specific therapies, including anti-EGFR treatment, have been clinically tested. The experience from the treatment of various types of cancers shows that patient outcome depends on the mutational status of particular molecules, including epithelial growth factor receptor (EGFR), Kirsten rat sarcoma viral oncogene homolog (KRAS), v-Raf murine sarcoma viral oncogene homolog B1 (BRAF) and phosphatidylinositol4,5-bisphosphate 3-kinase, catalytic subunit $\alpha$ (PIK3CA). Therefore, the aim of the present study was to assess the occurrence and potential use of these markers in patients with meningioma. In total, 55 formalin-fixed, paraffin-embedded meningioma samples were subjected to genomic sequencing of $E G F R$ (exons 18-21), KRAS (exon 1), BRAF (exon 15) and PI3K (exons 9, 20). No mutations were identified in $E G F R$, $K R A S$ or $B R A F$. Point mutations in PIK3CA were revealed in the samples of two patients with atypical and anaplastic meningiomas. Although these mutations appear to be rare, this result, along with previously reported findings, indicates that the $\mathrm{PI} 3 \mathrm{~K} /$ protein kinase B pathway may serve as a more reasonable molecular target for meningioma than EGFR.
\end{abstract}

\section{Introduction}

Meningiomas are one of the most frequent intracranial tumors. The majority of meningiomas are benign lesions of World Health Organization (WHO) grade I (GI), however, certain

Correspondence to: Dr Mateusz Bujko, Department of Molecular and Translational Oncology, Maria Sklodowska-Curie Memorial Cancer Center and Institute of Oncology, 5 W.K. Roentgena, Warsaw 02-781, Poland

E-mail: mbujko@coi.waw.pl

Key words: meningioma, EGFR, PI3K, PIK3CA, mutations histopathological variants are associated with more aggressive clinical behavior and correspond to WHO GII and III (1). The GII and III meningiomas have an increased risk of local recurrence. The standard treatment for high-grade meningiomas involves surgical resection with optional radiotherapy, and radiosurgery in the case of an incomplete resection. No pharmacological agents are routinely used for the treatment of meningiomas. The epithelial growth factor receptor (EGFR) is one of the most extensively studied molecular targets for anti-cancer therapy and is also considered as a target for meningioma treatment (2). This receptor is expressed in most meningioma patients and was initially identified to be involved in tumor development (3). The results of studies on EGFR expression in meningiomas are not entirely consistent. The percentage of EGFR-immunopositive tumors range between $50 \%(\mathrm{n}=85)(4)$ and $89 \%(\mathrm{n}=132)(5)$. In addition to the expression status, receptor activation has also been shown in patients $(6,7)$. In the IOMM-Lee meningioma cell line, the EGFR pathway was shown to be involved in radiation-induced progression (8). EGFR activates several downstream pathways, primarily those of mitogen-activated protein kinase (MAPK), phosphatidylinositol-4,5-bisphosphate 3-kinase/protein kinase B (PI3K/AKT) and phospholipase $\mathrm{C}$, which have been found to play a role in meningioma pathogenesis $(7,9)$.

Based on this background, two of the low molecular weight anti-EGFR inhibitors, gefitinib and erlotinib, were introduced in phase II clinical trials in patients with recurrent meningioma (North American Brain Tumor Consortium Trials 00-01 and 01-03). In these studies, small groups of patients were included ( $n=9$ and $n=16$, respectively) and no clear effect of the treatment was observed (10).

The data from previous clinical trials on the treatment of other tumor types showed that clinical response may depend on the presence of the mutations in EGFR (11) and in genes encoding downstream proteins involved in the signal transduction, including Kirsten rat sarcoma viral oncogene homolog $(K R A S)$, v-Raf murine sarcoma viral oncogene homolog B1 $(B R A F)$ or phosphatidylinositol-4,5-bisphosphate 3-kinase, catalytic subunit $\alpha$ (PIK3CA) (12). Mutations of the EGFR tyrosine kinase (TK) domains lead to constitutive kinase activation and were shown to be a good predictive marker in non-small cell lung cancer, while KRAS and BRAF were negative prognostic markers in colorectal cancer $(12,13)$. 
PIK3CA encodes the catalytic subunit of the PI3K protein and is affected by gain of function mutations in a significant ratio of solid tumors (14). The role of PIK3CA mutations in anti-EGFR therapy has not been definitively clarified. In colorectal cancer they coexist with KRAS mutations and are considered to be co-responsible for the resistance to targeted therapy (12). The mutations in the aforementioned downstream proteins generally cause activation of the MAPK or AKT pathways independent of the ligand binding or receptor status, and in lung cancer they have been shown to be mutually exclusive with EGFR mutations (15).

Previous meningioma studies have shown that EGFR does not undergo gene amplification $(16,17)$, but to the best of our knowledge the point mutations in the TK domain of the receptor, which is encoded by exons 18-21, have not been analyzed. Each of the BRAF, KRAS and PI3K genes has been analyzed once previously (18-20). The present study aimed to assess the occurrence of the mutations in the EGFR TK domain and in the selected downstream genes, KRAS, $B R A F$ and $P I K 3 C A$, in a relatively large group of meningioma patients.

\section{Materials and methods}

Patients and tissue samples. Tissue samples used for DNA isolation and genomic sequencing consisted of archival formalin-fixed, paraffin-embedded (FFPE) meningioma specimens from patients who underwent surgical tumor resection at the Department of Neurosurgery, M. Sklodowska-Curie Memorial Oncology Center (Warsaw, Poland) between 2007 and 2012. In total, 55 meningioma samples, including 20 GI,22 GII and 13 GIII, were used in the study. All tissue samples underwent histopathological examination that involved a review of the initial diagnosis and grading according to the 2007 WHO guidelines (1). The representative tissue samples were selected for molecular analysis. Patient characteristics are listed in Table I. Written informed consent was obtained from the patients and the study was approved by the local ethics committee of M. Sklodowska-Curie Memorial Cancer Center and Institute of Oncology (Warsaw, Poland).

Identification of genomic mutations. FFPE tissue samples were manually macrodissected and DNA was isolated with the use of a QIAamp DNA mini kit (Qiagen, Hilden, Germany). The purity and concentration of the DNA samples were assessed with the use of NanoDrop 1000 spectrophotometer (Thermo Scientific, Rockford, IL, USA) and with PicoGreen dsDNA quantitation assay (Invitrogen Life Technologies, Carlsbad, CA, USA).

DNA was amplified by polymerase chain reaction (PCR) containing 1X PCR buffer, $2 \mathrm{mM} \mathrm{MgCl} \mathrm{Mg}_{2}, 250 \mu \mathrm{M}$ of each oligonucleotide, $0.15 \mu \mathrm{M}$ of each PCR primer and 0.5 units of FastStart Taq DNA Polymerase (Roche Diagnostics, Mannheim, Germany). The GeneAmp 9700 PCR system (Applied Biosystems, Foster City, CA, USA) was used with the following cycling conditions: Initial denaturation at $94^{\circ} \mathrm{C}$ for $3 \mathrm{~min}$, followed by 40 cycles of $30 \mathrm{sec}$ at $94^{\circ} \mathrm{C}, 30 \mathrm{sec}$ at $57^{\circ} \mathrm{C}$ and $30 \mathrm{sec}$ at $72^{\circ} \mathrm{C}$, and a final elongation for $7 \mathrm{~min}$ at $72^{\circ} \mathrm{C}$. The PCR products were analyzed by electrophoresis in a $2 \%$ agarose gel, visualized with the use of ethidium bromide
Table I. Patient characteristics.

\begin{tabular}{lc}
\hline Characteristics & Value \\
\hline Patients, n & 55 \\
Male & 21 \\
Female & 34 \\
Age, years & \\
Range & $21-80$ \\
Median & 55 \\
Grade, n & \\
GI & 30 \\
Meningioma menigotheliale & 7 \\
Meningioma fibroblasticum & 4 \\
Meningioma transitionale & 13 \\
Other & 6 \\
GII meningioma atypicum & 16 \\
GIII meningioma anaplasticum & 9
\end{tabular}

GI, grade I.

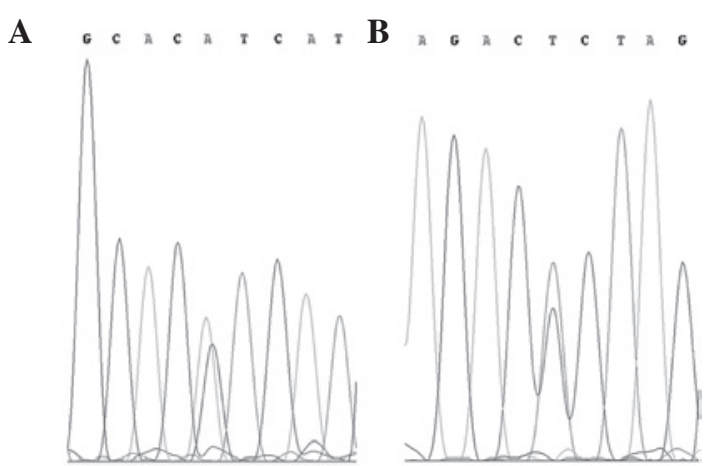

Figure 1. Genomic sequencing electropherogram of the samples with PIK3CA mutations. (A) GII meningioma sample with an $\mathrm{A}>\mathrm{AG}, 1047 \mathrm{H}>\mathrm{H} / \mathrm{R}$ mutation in codon 3140. (B) GIII meningioma sample with a $\mathrm{C}>\mathrm{CT}, 1025 \mathrm{~T}>\mathrm{T}$ silent mutation in codon 3075. PIK3CA, phosphatidylinositol-4,5-bisphosphate 3-kinase, catalytic subunit $\alpha$; GII, grade II.

and subjected to DNA sequencing. Briefly, $2.5 \mu \mathrm{l}$ of the PCR product was purified with the use of ExoStar (GE Healthcare Life Sciences, Pittsburgh, PA, USA) and labeled with BigDye Terminator v.3.1 (Applied Biosytems) according to the manufacturer's instructions. The ABI PRISM 3300 Genetic Analyzer (Applied Biosystems) was used for capillary electrophoresis and the sequence readout.

\section{Results}

All the DNA regions involved in the sequence analysis (EGFR exons 18-21, KRAS exon 1, BRAF exon 15 and PI3K exons 9 and 20) were successfully amplified and sequenced for all the patients enrolled. No mutations were identified in either EGFR, KRAS or BRAF. There were two PIK3CA exon 20 mutations identified. These were the missense mutation $\mathrm{A}>\mathrm{AG}, 1047 \mathrm{H}>\mathrm{H} / \mathrm{R}$ in codon 3140 , and the silent mutation $\mathrm{C}>\mathrm{CT}, 1025 \mathrm{~T}>\mathrm{T}$ in codon 3075 (Fig. 1). These samples were resected from a 54-year-old male patient with a GII atypical meningioma in the cerebellopontine angle and from a 
53-year-old female patient with a GIII anaplastic meningioma located in the cerebellopontine angle, respectively.

\section{Discussion}

The results of the present study are consistent with previously reported studies that also did not identify an incidence of mutations in KRAS (17) or BRAF (18). The lack of mutations in the EGFR TK domain and the downstream proteins that have been observed may indicate a low importance of this pathway in the growth of meningiomas, despite a previously suggested hypothesis (6). This is in line with previous studies that report no incidence of $E G F R$ gene amplification in meningiomas (16), as these molecular aberrations have a similar effect on signal transduction. This is also consistent with a previous study reporting that EGFR expression occurs less frequently in malignant meningiomas than in GI patients (5), and EGFR-positive patients have an improved survival prognosis compared with EGFR-negative patients (4).

The purpose of the mutation screening in the present study was to assess whether it is possible to use the molecular background to identify meningioma patients who may have a potentially improved response to anti-EGFR treatment, as is possible in the case of other tumors. The results show that known predictive markers have no value in meningiomas, and the lack of EGFR and downstream protein mutations may partially explain the results of the clinical trials with the anti-EGFR inhibitors, erlotinib and gefitinib (10).

All the aforementioned data indicate that despite the fact that EGFR is expressed in meningeal tumors, it does not contribute to tumor development or progression, as was hypothesized initially (9). This in turn indicates that the EGFR pathway is not a promising target for the treatment of meningiomas.

In the present study, the only identified genetic aberrations were the mutations of the kinase domain of PIK3CA that were observed in two patients with GII and GIII tumors. The genetic changes, $1047 \mathrm{H}>\mathrm{H} / \mathrm{R}$ and $1025 \mathrm{~T}>\mathrm{T}$, have already been reported in the COSMIC (Catalog of Somatic Mutations in Cancer) database (database ID 775 and 21451, respectively). The $1047 \mathrm{H}>\mathrm{H} / \mathrm{R}$ missense mutation is the most common mutation reported in tumor samples and accounts for $24.4 \%$ of all variations of the PIK3CA coding sequence submitted to the COSMIC database. This mutation has revealed strong oncogenic potential in vivo (20) and recently was also shown to be associated with the response to PI3K/AKT pathway inhibitors in clinical trials (21). The second identified genomic variation does not affect the amino acid sequence and is less frequent in cancer patients, with a frequency of $0.3 \%$ PIK3CA mutations reported in the COSMIC database.

The occurrence of the PIK3CA mutations in meningiomas was investigated in a previous study, and one of the patients with anaplastic meningioma was identified as a mutation carrier (19). The combination of data from the previous and current studies indicates a 3.4\% (3/87) frequency of PIK3CA variations in atypical and anaplastic meningiomas. This can be described as rare, but the PI3K/AKT pathway may also be deregulated by the mutations in phosphatase and tensin homolog (PTEN); a PI3K inhibitor. In meningiomas, these gene mutations have previously been shown to occur with a frequency similar to the PIK3CA mutations (22). In total, two of the PTEN mutations were identified in GIII tumors and one in a radiation-induced meningioma. No PIK3CA or PTEN mutations were found in any GI patients $(19,22)$. This indicates the involvement of this pathway in tumor progression. The phosphorylation in the PI3K/AKT pathway was previously observed as being more frequent in GII and III meningiomas, and pharmacological inhibition of this pathway in primary malignant meningioma cells resulted in reduced proliferation and survival (7).

In the light of all the presented facts it appears that PI3K and its co-regulators may be more reasonable molecular targets in meningiomas than EGFR. Recently, a number of small molecular inhibitors that target PI3K have been reported in clinical trials (21), and mutations of PIK3CA/PTEN have been show to have a predictive value in the administration of these compounds $(23,24)$. We believe that these results should encourage clinicians to consider the possible use of this pharmacological treatment in malignant meningiomas.

\section{Acknowledgements}

This study was supported by the National Science Centre (grant no. UMO-2011/01/D/NZ5/02798).

\section{References}

1. Louis DN, Ohgaki H, Wiestler OD, et al: The 2007 WHO classification of tumours of the central nervous system. Acta Neuropathol 114: 97-109, 2007.

2. Normanno N, De Luca A, Bianco C, et al: Epidermal growth factor receptor (EGFR) signaling in cancer. Gene 366: 2-16, 2006.

3. Torp SH, Helseth E, Dalen A and Unsgaard G: Expression of epidermal growth factor receptor in human meningiomas and meningeal tissue. APMIS 100: 797-802, 1992.

4. Smith JS, Lal A, Harmon-Smith M, Bollen AW and McDermott MW: Association between absence of epidermal growth factor receptor immunoreactivity and poor prognosis in patients with atypical meningioma. J Neurosurg 106: 1034-1040, 2007.

5. Wernicke AG, Dicker AP, Whiton M, et al: Assessment of epidermal growth factor receptor (EGFR) expression in human meningioma. Radiat Oncol 5: 46, 2010.

6. Carroll RS, Black PM, Zhang J, Kirsch M, Percec I, Lau N and Guha A: Expression and activation of epidermal growth factor receptors in meningiomas. J Neurosurg 87: 315-323, 1997.

7. Mawrin C, Sasse T, Kirches E, et al: Different activation of mitogen-activated protein kinase and Akt signaling is associated with aggressive phenotype of human meningiomas. Clin Cancer Res 11: 4074-4082, 2005.

8. Kargiotis O, Chetty C, Gogineni V, et al: uPA/uPAR downregulation inhibits radiation-induced migration, invasion and angiogenesis in IOMM-Lee meningioma cells and decreases tumor growth in vivo. Int J Oncol 33: 937-947, 2008.

9. Johnson M and Toms S: Mitogenic signal transduction pathways in meningiomas: novel targets for meningioma chemotherapy? J Neuropathol Exp Neurol 64: 1029-1036, 2005.

10. Norden AD, Raizer JJ, Abrey LE, et al: Phase II trials of erlotinib or gefitinib in patients with recurrent meningioma. J Neurooncol 96: 211-217, 2010.

11. Lynch TJ, Bell DW, Sordella R, et al: Activating mutations in the epidermal growth factor receptor underlying responsiveness of non-small-cell lung cancer to gefitinib. N Engl J Med 350: 2129-2139, 2004

12. De Roock W, Claes B, Bernasconi D, et al: Effects of KRAS, BRAF, NRAS, and PIK3CA mutations on the efficacy of cetuximab plus chemotherapy in chemotherapy-refractory metastatic colorectal cancer: a retrospective consortium analysis. Lancet Oncol 11: 753-762, 2010. 
13. Brand TM, Iida M and Wheeler DL: Molecular mechanisms of resistance to the EGFR monoclonal antibody cetuximab. Cancer Biol Ther 11: 777-792, 2011.

14. Samuels Y, Wang Z, Bardelli A, et al: High frequency of mutations of the PIK3CA gene in human cancers. Science 304: 554,2004

15. Kosaka T, Yatabe Y, Endoh H, Kuwano H, Takahashi T and Mitsudomi T: Mutations of the epidermal growth factor receptor gene in lung cancer: biological and clinical implications. Cancer Res 64: 8919-8923, 2004

16. Diedrich U, Lucius J, Baron E, Behnke J, Pabst B and Zoll B: Distribution of epidermal growth factor receptor gene amplification in brain tumours and correlation to prognosis. J Neurol 242: 683-688, 1995.

17. Chaffanet M, Chauvin C, Lainé M, et al: Comparative analysis of the NF2, TP53, PTEN, KRAS, NRAS and HRAS genes in sporadic and radiation-induced human meningiomas. Int J Cancer 94: 218-221, 2001.

18. Schindler G, Capper D, Meyer J, et al: Analysis of BRAF V600E mutation in 1,320 nervous system tumors reveals high mutation frequencies in pleomorphic xanthoastrocytoma, ganglioglioma and extra-cerebellar pilocytic astrocytoma. Acta Neuropathol 121: 397-405, 2011.
19. Pang JC, Chung NY, Chan NH, Poon WS, Thomas T and $\mathrm{Ng}$ HK: Rare mutation of PIK3CA in meningiomas. Acta Neuropathol 111: 284-285, 2006

20. Bader AG, Kang S and Vogt PK: Cancer-specific mutations in PIK3CA are oncogenic in vivo. Proc Natl Acad Sci USA 103: 1475-1479, 2006.

21. Janku F, Wheler JJ, Naing A, et al: PIK3CA mutation H1047R is associated with response to PI3K/AKT/mTOR signaling pathway inhibitors in early-phase clinical trials. Cancer Res 73: 276-284, 2013.

22. Peters N, Wellenreuther R, Rollbrocker B, Hayashi Y, Meyer-Puttlitz B, Duerr EM, Lenartz D, Marsh DJ, Schramm J, Wiestler OD, Parsons R, Eng C and von Deimling A: Analysis of the PTEN gene in human meningiomas. Neuropathol Appl Neurobiol 24: 3-8, 1998

23. Willems L, Tamburini J, Chapuis N, Lacombe C, Mayeux P and Bouscary D: PI3K and mTOR signaling pathways in cancer: new data on targeted therapies. Curr Oncol Rep 14: 129-138, 2012.

24. Martini M, Ciraolo E, Gulluni F and Hirsch E: Targeting PI3K in cancer: Any good news? Front Oncology 3: 108, 2013. 